\title{
Destruction of chemical warfare agents using metal-organic frameworks
}

\author{
Joseph E. Mondloch ${ }^{1 \dagger}$, Michael J. Katz ${ }^{1 \dagger}$, William C. Isley $\mathrm{III}^{2}$, Pritha Ghosh ${ }^{3}$, Peilin Liao ${ }^{3}$, \\ Wojciech Bury ${ }^{1}$, George W. Wagner ${ }^{4}$, Morgan G. Hall ${ }^{4}$, Jared B. DeCoste ${ }^{5}$, Gregory W. Peterson ${ }^{4}$, \\ Randall Q. Snurr ${ }^{3}$, Christopher J. Cramer ${ }^{2}$, Joseph T. Hupp ${ }^{1 \star}$ and Omar K. Farha ${ }^{1,6 \star}$
}

\begin{abstract}
Chemical warfare agents containing phosphonate ester bonds are among the most toxic chemicals known to mankind'. Recent global military events, such as the conflict and disarmament in Syria ${ }^{2}$, have brought into focus the need to find effective strategies for the rapid destruction of these banned chemicals. Solutions are needed for immediate personal protection (for example, the filtration and catalytic destruction of airborne versions of agents), bulk destruction of chemical weapon stockpiles, protection (via coating) of clothing, equipment and buildings, and containment of agent spills ${ }^{3}$. Solid heterogeneous materials such as modified activated carbon or metal oxides exhibit many desirable characteristics for the destruction of chemical warfare agents ${ }^{4-6}$. However, low sorptive capacities, low effective active site loadings, deactivation of the active site, slow degradation kinetics, and/or a lack of tailorability offer significant room for improvement in these materials. Here, we report a carefully chosen metal-organic framework (MOF) material featuring high porosity and exceptional chemical stability that is extraordinarily effective for the degradation of nerve agents and their simulants. Experimental and computational evidence points to Lewis-acidic $\mathbf{Z r}^{\mathrm{IV}}$ ions as the active sites and to their superb accessibility as a defining element of their efficacy.
\end{abstract}

MOFs constitute a remarkably broad, and very rapidly growing, class of crystalline materials (Fig. 1); at their simplest, they comprise uniformly arrayed metal-containing nodes separated by organic linkers ${ }^{7}$. Their exceptional surface areas as well as high porosity, their amenability to modular design and construction, and their ability to present high concentrations of metal ions (or clusters) dispersed in structurally well-defined matrices make MOFs singularly attractive as solid sorbents and heterogeneous catalysts ${ }^{8}$. In this regard, we, and others, have demonstrated that the nodes of selected MOFs can catalytically hydrolyse phosphoester-containing compounds ${ }^{9}$, a key step in the degradation of many chemical warfare agents. For example, using the well-known twelve-connected $\mathrm{Zr}_{6}$-based MOF UiO-66, we were able to catalytically hydrolyse the nerve agent simulant dimethyl 4-nitrophenyl phosphate (DMNP, see Fig. 2a $)^{10}$. Given their known outstanding thermal ${ }^{11}$, mechanical ${ }^{12}$ and hydrolytic stabilities ${ }^{13}$, MOFs containing $\mathrm{Zr}_{6}$ nodes and multitopic carboxylated linkers are among the most promising classes of potentially catalytic materials for deployment in personal chemical protection. Further motivating our earlier selection of UiO-66 was its presentation of multiple $\mathrm{Zr}-\mathrm{OH}-\mathrm{Zr}$ moieties-potential mimics of the Lewis-acidic $\mathrm{Zn}-\mathrm{OH}-\mathrm{Zn}$ active site found in $\mathrm{G}$-agent destroying enzymes such as phosphotriesterase. Despite the success of these initial studies, so far MOFs have exhibited unacceptably slow kinetics for capture and destruction of nerve agents and their simulants 9 .

A key issue in exploiting MOFs for the hydrolysis of chemical warfare agents has been that their pore apertures have been too small to admit agents to the interior spaces where the vast majority of potential active sites are located. In other words, nearly all of the sorptive capacity and resultant hydrolysis activity was restricted to sites on the exterior of the MOF crystallites (presumably at missing-linker defect sites). A related issue, specifically with $\mathrm{UiO}-66$, is that the most desirable reaction sites are already occupied by linkers. To overcome these challenges we turned our attention towards the recently reported $\mathrm{Zr}_{6}$-based MOF NU-1000 (Fig. 1) $)^{14}$. NU-1000 is built up from eight (rather than twelve) connected $\mathrm{Zr}_{6}\left(\mu_{3}-\mathrm{O}\right)_{4}\left(\mu_{3}-\mathrm{OH}\right)_{4}\left(\mathrm{H}_{2} \mathrm{O}\right)_{4}(\mathrm{OH})_{4}$ nodes and tetratopic $1,3,6,8$ ( $p$-benzoate) pyrene linkers (TBAPy ${ }^{4-} ;$ ref. 15$)$. The resulting structure directs a total of four terminal-zirconium-ligated aquo and hydroxo groups per node into exceptionally wide $(31 \AA)$ channels, and a further four into smaller $10 \AA$ channels (versus zero for defect-free UiO-66). The ultrawide channels allow bulky phosphate ester molecules to permeate the entire framework, including the vast MOF interior. Here we demonstrate that NU-1000 is highly active for the destruction of the nerve agent simulant DMNP and, more importantly, the highly toxic chemical warfare agent GD (O-pinacolyl methylphosphonofluoridate, also known as Soman).

To probe the efficacy of NU-1000 for the catalytic destruction of phosphate ester bonds we first investigated the simulant DMNP. Nerve agent simulants are an essential tool and precautionary measure for assessing phosphate ester decomposition in nearly all laboratories, given the highly toxic nature of chemical warfare agents. Simulants (such as DMNP) also allow rigorous physical characterization as well as mechanistic investigations of key catalytic systems that can help drive the development of superior decontamination catalysts. Catalytic hydrolysis of DMNP was carried out using NU-1000 in an aqueous $\mathrm{N}$-ethylmorpholine buffered solution at pH 10 (Fig. 2a). Hydrolysis of the phosphorus nitrophenyl bond was observed by ${ }^{31} \mathrm{P}$ NMR spectroscopy (Supplementary Fig. 3). The kinetics of the hydrolysis reaction

\footnotetext{
${ }^{1}$ Department of Chemistry, Northwestern University, 2145 Sheridan Road, Evanston, Illinois 60208, USA. ${ }^{2}$ Department of Chemistry, Supercomputing Institute, and Chemical Theory Center, University of Minnesota, Minneapolis, Minnesota 55455, USA. ${ }^{3}$ Department of Chemical \& Biological Engineering, Northwestern University, 2145 Sheridan Road, Evanston, Illinois 60208, USA. ${ }^{4}$ Edgewood Chemical Biological Center, US Army Research, Development, and Engineering Command, 5183 Blackhawk Road, Aberdeen Proving Ground, Maryland 21010, USA. ${ }^{5}$ Leidos Inc., PO Box 68 Gunpowder, Maryland 21010 , USA. ${ }^{6}$ Department of Chemistry, Faculty of Science, King Abdulaziz University, Jeddah 21589, Saudi Arabia. These authors contributed equally to this work. *e-mail: j-hupp@northwestern.edu; o-farha@northwestern.edu
} 

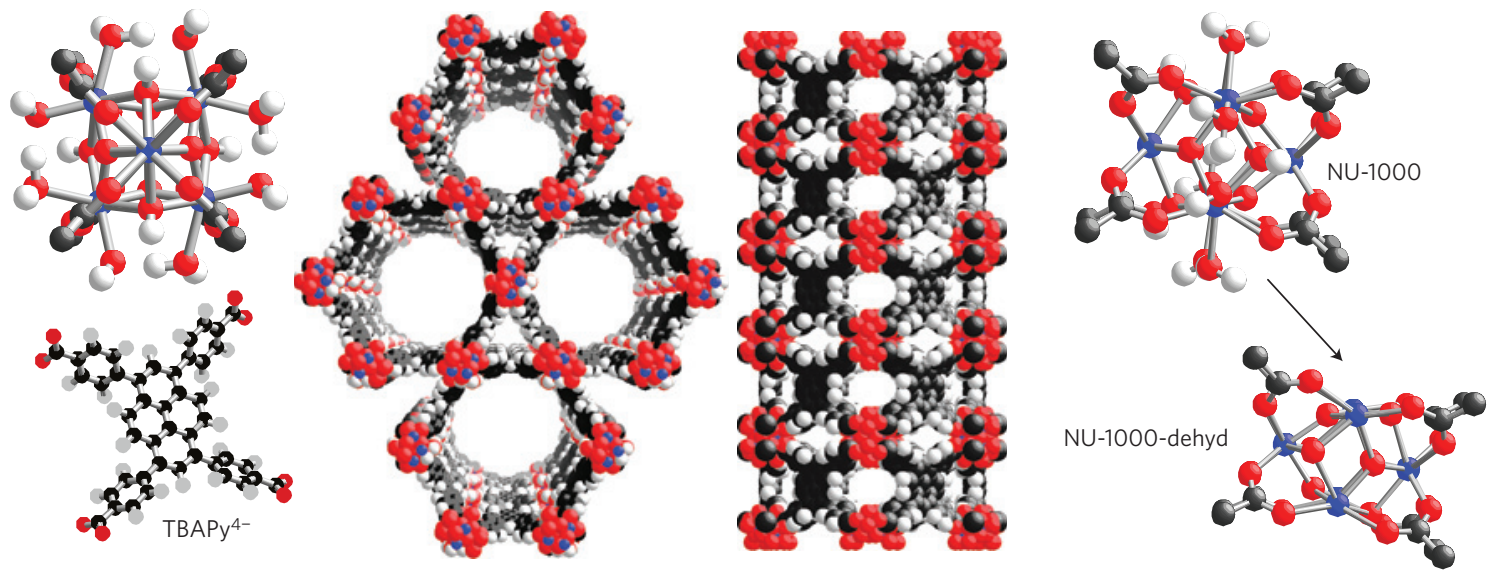

Figure 1 | Molecular representations of the NU-1000 node and linker (left), MOF topology (two views, centre), and the dehydration of the NU-1000 node (right). Colour code: $\mathrm{Zr}$ (blue); O (red); C (black); H (white).

Table 1 | Comparison of phosphate ester (top) and phosphatefluoride bond (bottom) degradation by MOFs.

\begin{tabular}{llll} 
MOF & Substrate* $^{*}$ & $\boldsymbol{t}_{1 / 2}(\mathbf{m i n})$ & Ref. \\
\hline NU-1000-dehyd & Simulants & & \\
NU-1000 & DMNP & 1.5 & This work \\
UiO-66 & DMNP & 15 & This work \\
MIL-101(Cr)-DAAP $^{\dagger}$ & DMNP & 45 & 10 \\
$\left\{\left[\mathrm{Ho}_{4}(\mathrm{dpdo})_{8}\left(\mathrm{H}_{2} \mathrm{O}\right)_{16}\right.\right.$ & DENP & 300 & 20 \\
$\left.\left.\mathrm{BiW}_{12} \mathrm{O}_{40}\right]\left(\mathrm{H}_{2} \mathrm{O}\right)_{2}\right\}^{7+}$ & & 295,000 & 22 \\
& Chemical warfare agents & \\
NU-1000 & GD & 3 & This work \\
NU-1000 & GD & 36 & This work \\
HKUST-1 & GD & 2,880 & 21 \\
\hline
\end{tabular}

*Dimethyl 4-nitrophenyl phosphate (DMNP); diethyl 4-nitrophenyl phosphate (DENP); bis(4-nitrophenyl) phosphate (BNPP); ‘dialkylaminopyridine (DAAP); ${ }^{\ddagger}$ Run in aqueous solution in the presence of $\mathrm{N}$-ethylmorpholine buffer; ${ }^{*}$ Run under $50 \%$ relative humidity.

were followed by monitoring the formation of $p$-nitrophenoxide via visible-region absorption spectroscopy $\left(\lambda_{\max }=407 \mathrm{~nm}\right)$; see the example in Fig. 2b. It is notable that the $p$-nitrophenoxide anion forms exclusively at $\mathrm{pH} 10$. We observed $77 \%$ conversion over the course of $60 \mathrm{~min}$ (Fig. 2c, red circles), and the measured half-life (that is, $50 \%$ conversion) was found to be $15 \mathrm{~min}$. This corresponds to an initial turnover frequency (TOF) of $0.06 \mathrm{~s}^{-1}$, assuming the entire framework is accessible to DMNP (further details regarding the calculation of TOFs are reported in the Supplementary Information). The observed half-life represents the fastest known decomposition of a phosphate ester nerve agent simulant by a MOF so far (see Table 1). Notably the background reaction is negligible over the same time course $(3 \%$ conversion after $60 \mathrm{~min}$; see Fig. 2c, black diamonds). Finally NU-1000 is completely recyclable for the hydrolysis of DMNP over three runs (Supplementary Fig. 6).

To establish that the observed catalysis is indeed heterogeneous, we filtered NU-1000 from the reaction mixture and resumed monitoring the formation of the $p$-nitrophenoxide anion. No further conversion was observed (Supplementary Fig. 4). Powder $\mathrm{X}$-ray diffraction (PXRD) measurements showed that NU-1000 remains crystalline after service as a catalyst (Supplementary Fig. 5). We also investigated a soluble, molecular, zirconium-based cluster, $\mathrm{Zr}_{6}(\mathrm{O})_{4}(\mathrm{OH})_{4}(\mathrm{BzA})_{12}$ (see ref. 16); over the course of $60 \mathrm{~min}$ the hydrolysis reaction goes only towards $20 \%$ completion
(Supplementary Fig. 7). Clearly, soluble homogeneous $\mathrm{Zr}_{6}$-based clusters are not as potent as the corresponding site-isolated, eightconnected clusters within the highly porous NU-1000 scaffold. As a further control, we examined $\mathrm{Zr}(\mathrm{OH})_{4}$, which has been shown to be effective for the destruction of phosphate-based nerve agents ${ }^{6}$. Under our experimental conditions, the hydrolysis of DMNP by $\mathrm{Zr}(\mathrm{OH})_{4}$ is negligible (Supplementary Fig. 8). Overall, our results demonstrate the heterogeneous nature of NU-1000, as well as the advantages of presenting $\mathrm{Zr}_{6}$-based clusters within a highly porous network for the catalytic hydrolysis of phosphoester bonds.

To gain insight into the potential mechanism(s) of DMNP hydrolysis in NU-1000 we turned to quantum chemical calculations based on density functional theory (DFT). We found two classes of favourable binding modes for DMNP at the $\mathrm{Zr}_{6}\left(\mu_{3}-\mathrm{O}\right)_{4}\left(\mu_{3}-\mathrm{OH}\right)_{4}\left(\mathrm{H}_{2} \mathrm{O}\right)_{4}(\mathrm{OH})_{4}$ node of NU-1000. In the first, DMNP forms hydrogen bonds with the node-ligated water and hydroxide moieties. An example is shown in Fig. 3a, with a full list of investigated binding modes given in Supplementary Table 1. The lowest-energy configuration has a binding free energy $\left(\Delta G_{\text {assoc }}^{\circ}\right)$ of $-26 \mathrm{~kJ} \mathrm{~mol}^{-1}$ and in addition to hydrogen bonding is stabilized by $\pi-\pi$ stacking interactions between the phenyl ring of DMNP and the benzene ring of the TBAPy ${ }^{4-}$ linkers from NU-1000 (not shown for clarity in Fig. 3). The $\pi-\pi$ stacking interactions occur at $3.1 \AA$, which is near the optimal distance for $\pi-\pi$ association ${ }^{17}$.

Next we removed a terminal water molecule from the node of NU-1000 to simulate ligand dissociation followed by DMNP substrate binding (Supplementary Tables 2 and 3). Here DMNP was able to directly interact with Lewis-acidic $\mathrm{Zr}^{\mathrm{IV}}$ sites in the $\mathrm{Zr}_{6}$ cluster of NU-1000. The electrostatic interaction between the $\mathrm{P}=\mathrm{O}$ moiety of DMNP and $\mathrm{Zr}$ takes precedence, whereas the $\pi-\pi$ stacking interactions between the linker and DMNP are no longer present. This most stable DMNP- $\mathrm{Zr}^{\mathrm{IV}}$ coordinated configuration is $22 \mathrm{~kJ} \mathrm{~mol}^{-1}$ uphill in free energy relative to the separated agent and NU-1000; its geometry includes hydrogen-bonding interactions between an $-\mathrm{OCH}_{3}$ of DMNP and a $\mu_{3}-\mathrm{O}$ of the NU-1000 node. The strong propensity for catalysis is driven by the stability of the hydrolysed product, which is $48 \mathrm{~kJ} \mathrm{~mol}^{-1}$ downhill in free energy, again relative to separated reactants (Supplementary Table 5). Remarkably, our simulations are very similar to those calculated for the interaction and hydrolysis of DMNP by the active site of the phosphotriesterase enzyme ${ }^{18}$. In the enzyme, hydrolysis (from a bound $\mathrm{Zn}^{2+}$ centre) is approximately $52.9 \mathrm{~kJ} \mathrm{~mol}^{-1}$ downhill. Furthermore, the optimized $\mathrm{Zn}-\mathrm{O}=\mathrm{P}$ distances are $2.24 \AA$ and $1.50 \AA$-close to the $\mathrm{Zr}-\mathrm{O}=\mathrm{P}$ distances in our simulations of $2.22 \AA$ and $1.49 \AA$ respectively ${ }^{19}$.

To experimentally probe the Lewis-acid activation pathway, we thermally treated NU-1000 at $300^{\circ} \mathrm{C}$ to remove terminal aquo 

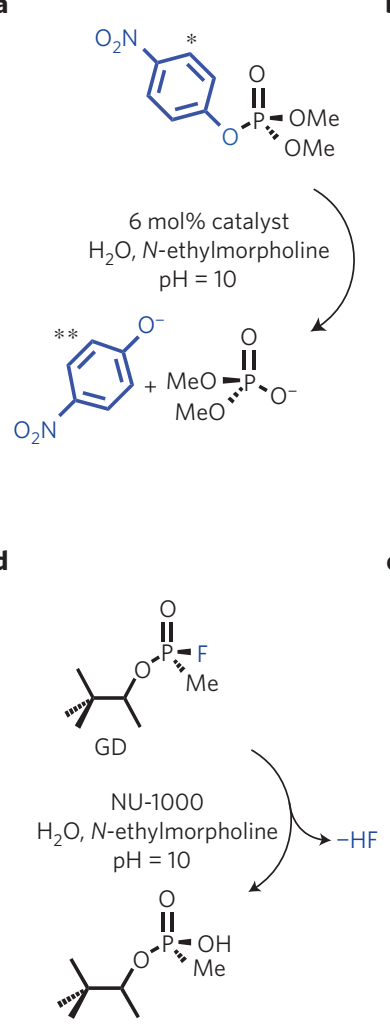

b

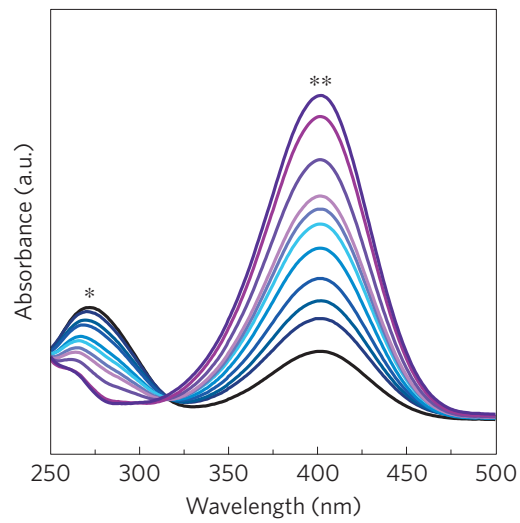

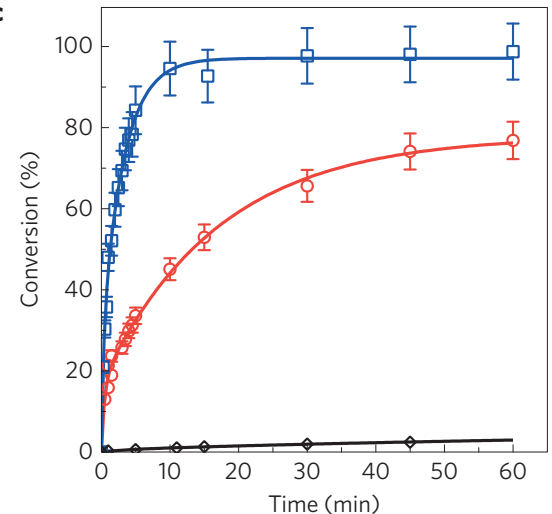

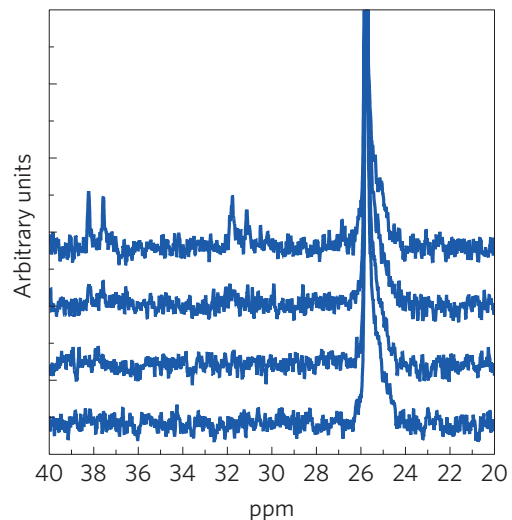

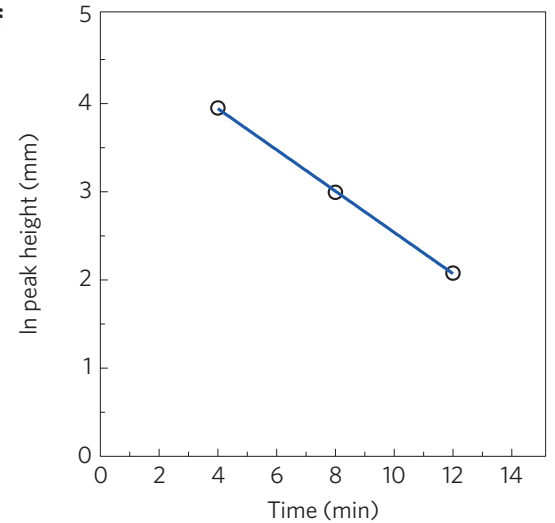

Figure $\mathbf{2}$ | Hydrolysis data and reactions for DMNP and GD. a, Reaction conditions for the catalytic decomposition of DMNP using NU-1000. b. Ultraviolet-visible monitoring of the formation of $p$-nitrophenoxide. ${ }^{*}$ in $\mathbf{b}$ indicates the wavelength at which DMNP in a (top) absorbs and ${ }^{* *}$ indicates the absorbance of $p$-nitrophenoxide (blue molecule, bottom), signifying decomposition has occurred. c, Percentage conversion to $p$-nitrophenoxide versus time for the background reaction (black diamonds), NU-1000 (red circles) and NU-1000-dehyd (blue squares). The error bars indicate the standard deviation of three independent catalytic tests. $\mathbf{d}$, Reaction conditions for the decomposition of GD. e, ${ }^{31}$ P NMR spectra of GD. f, Loss of GD versus time monitored via ${ }^{31}$ P NMR spectroscopy.

and hydroxo ligands and convert bridging hydroxos to oxos (NU1000-dehyd, Fig. 1). It is well known in metal-oxide chemistry that thermal treatment removes coordinating $\mathrm{H}_{2} \mathrm{O}$ molecules as well as anionic hydroxide ligands (through condensation) to leave behind coordinatively unsaturated metal sites ${ }^{6}$. PXRD, diffuse reflectance infrared Fourier transform spectroscopy (DRIFTS) and thermogravimetric analysis (TGA) are all consistent with NU1000-dehyd having the molecular formula $\mathrm{Zr}_{6}\left(\mu_{3}-\mathrm{O}\right)_{8}(\mathrm{TBAPy})_{2}$ (Supplementary Figs 9-11). Structurally, on dehydration, four of the six $\mathrm{Zr}^{\mathrm{IV}}$ ions are six-coordinate, coordinatively unsaturated Lewisacidic ions (Fig. 1, NU-1000-dehyd). Thus, two-thirds of the metal ions within the NU-1000 platform are potentially available and functional as catalyst active sites, versus only a tiny percentage for either $\mathrm{UiO}-66$ or nanoparticulate $\mathrm{Zr}(\mathrm{OH})_{4}$. Under identical reaction conditions to those for NU-1000, NU-1000-dehyd is remarkably active for the hydrolysis of DMNP, yielding a half-life of only $1.5 \mathrm{~min}$ and exhibiting 100\% conversion after approximately $10 \mathrm{~min}$ (Fig. 2c, blue squares). This in turn yields an initial TOF of $0.11 \mathrm{~s}^{-1}$ for the hydrolysis of DMNP by NU-1000-dehyd. We find that the node does not re-hydrate to its original configuration over the time course of simulant hydrolysis. Further experiments indicate activity similar to that observed for NU-1000 is obtained when NU-1000-dehyd is exposed for two weeks or four months to ambient laboratory atmosphere (Supplementary Fig. 12). Like the parent material, NU1000-dehyd functions heterogeneously and remains crystalline after use as a catalyst (Supplementary Figs 16 and 17). Clearly, opening up $\mathrm{Zr}^{\mathrm{IV}}$ sites substantially enhances the hydrolysis of DMNP, data that is consistent with a mechanism involving $\mathrm{P}=\mathrm{O}$ binding at these sites as well as with the rate retardation that is predicted from theory for displacement of water from the hydrated node. These results are also supportive of our global hypothesis that selected MOFs may function as solid-state mimics of the well-known phosphotriesterase enzyme, which uses a pair of Lewis-acidic sites.

Because MOFs are crystallographically defined, we were able to carry out predictive simulations regarding the interactions of the simulant DMNP with the node of NU-1000. We also analysed computationally the binding of analogues of the chemical warfare agents GD and O-ethyl $S$-diisopropylaminoethyl methylphosphonothiolate (VX). In contrast to the simulations for DMNP, the hydrogenbonding interactions that were present are not thermodynamically favourable for the GD and VX analogues (for example, Fig. 3d; further information is given in Supplementary Table 6). We also find that the interactions of the $\mathrm{P}=\mathrm{O}$ moieties of the GD and VX analogues directly with the $\mathrm{Zr}^{\mathrm{IV}}$ ions are uphill in free energy, by 32 and $22 \mathrm{~kJ} \mathrm{~mol}^{-1}$, respectively. However, on coordinative binding, there are sizable driving forces for hydrolysis of the GD and VX analogues. Our simulations suggest selective hydrolysis of the P-F bond of GD is $83 \mathrm{~kJ} \mathrm{~mol}^{-1}$ downhill, whereas hydrolysis of the P-S bond of VX is $123 \mathrm{~kJ} \mathrm{~mol}^{-1}$ downhill and is predicted to be favoured over hydrolysis of $\mathrm{P}-\mathrm{O}$ at $79 \mathrm{~kJ} \mathrm{~mol}^{-1}$ downhill (further information is provided in the Supplementary Information).

Inspired by the catalysis results for DMNP, as well as the favourable thermodynamics uncovered computationally, we decided to see if NU-1000 could catalyse the hydrolysis of the chemical warfare agent GD. To start we used ${ }^{31} \mathrm{P}\left\{{ }^{1} \mathrm{H}\right\}$ NMR spectroscopy to investigate the decomposition of GD 
a

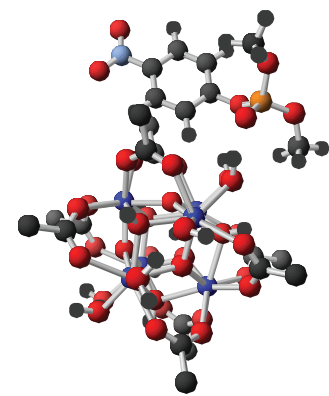

$\left(-26.4 \mathrm{~kJ} \mathrm{~mol}^{-1}\right)$

d

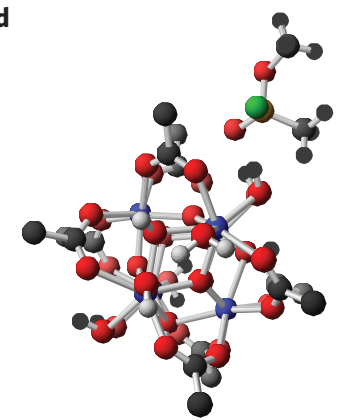

$\left(+26.8 \mathrm{~kJ} \mathrm{~mol}^{-1}\right)$

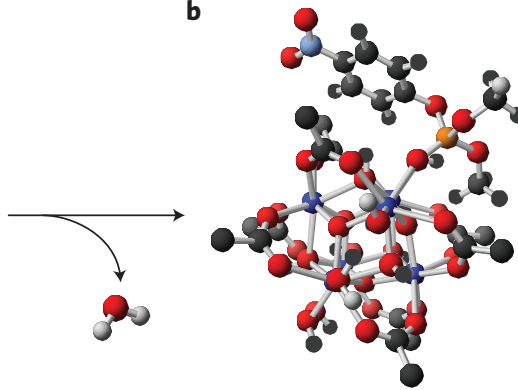

$\left(+21.8 \mathrm{~kJ} \mathrm{~mol}^{-1}\right)$

e

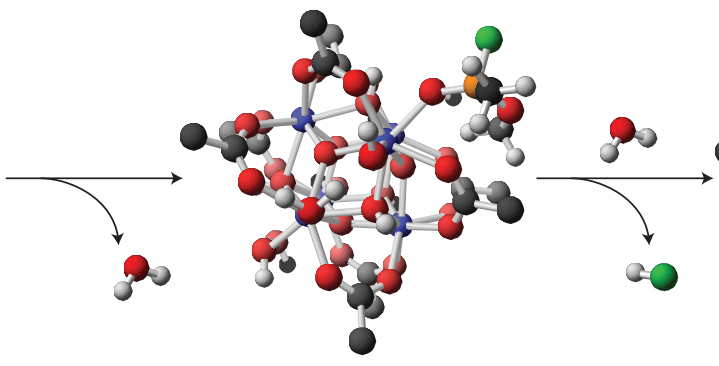

$\left(+32.2 \mathrm{~kJ} \mathrm{~mol}^{-1}\right)$ c

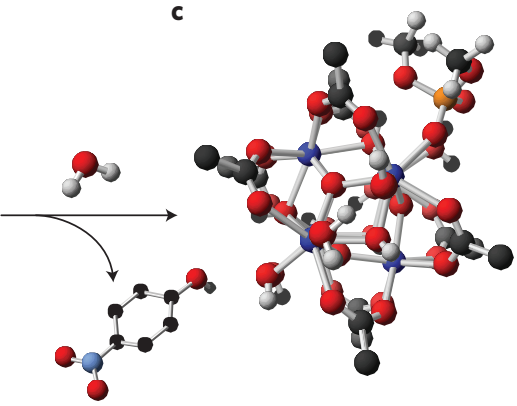

$\left(-48.1 \mathrm{~kJ} \mathrm{~mol}^{-1}\right)$

Figure 3 | Association and reaction energies predicted by DFT. a-f, Key $\Delta G_{\text {assoc }}^{\circ}(\mathbf{a}, \mathbf{d})$ and $\Delta G_{\mathrm{rxn}}^{\circ}(\mathbf{b}, \mathbf{c}, \mathbf{e}, \mathbf{f})$ values for the interaction of $D M N P$ and $G D$ analogue with the node of NU-1000. $\mathbf{a}$, DMNP binding. b. DMNP replacing a $\mathrm{H}_{2} \mathrm{O}$ molecule. c, Hydrolysis of DMNP. d, GD analogue binding. e, GD analogue replacing a $\mathrm{H}_{2} \mathrm{O}$ molecule. f, Hydrolysis of $\mathrm{GD}$. Colour code: $\mathrm{Zr}$ (blue); $\mathrm{O}$ (red); C (black); $\mathrm{H}$ (white); P (orange); N (light blue); F (green); $\mathrm{S}$ (yellow).

with NU-1000 in an $N$-ethylmorpholine buffered solution under conditions similar to those used for DMNP (Fig. 2d-f). Remarkably, the observed half-life was found to be just $3 \mathrm{~min}$ and the initial TOF was $0.013 \mathrm{~s}^{-1}$. The ${ }^{31} \mathrm{P}\left\{{ }^{1} \mathrm{H}\right\}$ NMR spectra are dominated by the presence of the product, pinacolyl methylphosphonic acid (PMPA) near $25 \mathrm{ppm}$, whereas the twin resonances for the GD isomers (near 31 and $38 \mathrm{ppm}$ ) are strongly depleted by the time we measure the initial data point. We also measured the decomposition of GD under $50 \%$ relative humidity using ${ }^{31} \mathrm{P}$ solid-state-NMR spectroscopy; the P-F coupled resonances at 26 and $34 \mathrm{ppm}$ disappear (Supplementary Fig. 19) ${ }^{6}$. Under these conditions NU1000 yields a reaction half-life of $36 \mathrm{~min}$ (Supplementary Fig. 19), nearly 80 times more active than the best MOF at present for the destruction of GD and comparable to the best existing solid-state materials for chemical threat protection (Table 1) ), $^{6,9}$. It is also worth noting that typically hundreds of milligrams of metal oxide are needed to observe similar decomposition rates for GD, whereas only thirty milligrams of NU-1000 was needed ${ }^{6}$.

In Table 1 (top), we have benchmarked NU-1000 and NU1000-dehyd versus other known MOFs that catalytically degrade phosphoester-containing compounds. NU-1000 and NU-1000dehyd represent the fastest known phosphoester-containing hydrolysis catalysts so far: 3 times and 30 times faster than found in our previous work with UiO-66 (ref. 10), and 20 times and 200 times faster than the well-known Cr-MIL-101, which contains coordinatively unsaturated $\mathrm{Cr}^{\text {III }}$ ions linked by benzene dicarboxylates ${ }^{20}$. NU1000 is also the fastest known MOF-based catalyst for the hydrolysis of GD (Table 1, bottom): 80-fold faster than the MOF HKUST-1 (under similar conditions and 960-fold faster in our buffered solution) which contains $\mathrm{Cu}^{\text {II }}$ paddlewheel-based nodes and trimesic acid linkers ${ }^{21}$. Finally, NU-1000 is among the most active solid heterogeneous catalysts known for the hydrolysis of GD (ref. 6). Our experimental and computational results suggest that the extraordinary activity of NU-1000 and NU-1000-dehyd is engendered by the unique eight-connected $\mathrm{Zr}_{6}\left(\mu_{3}-\mathrm{O}\right)_{4}\left(\mu_{3}-\mathrm{OH}\right)_{4}\left(\mathrm{H}_{2} \mathrm{O}\right)_{4}(\mathrm{OH})_{4}$ node and weak intermolecular interactions ( $\pi$-stacking for DMNP, more general attractive van der Waals interactions between linker and alkyl groups in GD or its analogue) that direct orientations between the substrate and catalyst-linker components, along with mesoporous channels that allow substrates access to highly Lewisacidic $\mathrm{Zr}^{\mathrm{IV}}$ sites. Given these exciting results, coupled with the highly modular nature of these unique solids and the ability to computationally select and experimentally install other metals and other potential substrate recognition sites, we anticipate further progress in using MOFs for the hydrolytic degradation of other chemical warfare agents (for example, VX) and their simulants and for the destruction of toxic industrial chemicals, which are potential improvised chemical threats.

\section{Methods}

Synthesis of NU-1000. $0.097 \mathrm{~g}$ of $\mathrm{ZrOCl}_{2} \cdot 8 \mathrm{H}_{2} \mathrm{O}(0.30 \mathrm{mmol})$ was weighed out into a 6 -dram vial and $2.7 \mathrm{~g}$ of benzoic acid was subsequently added. The solids were dissolved via sonication in $8 \mathrm{ml}$ of DMF and placed in an oven at $80^{\circ} \mathrm{C}$ for $1 \mathrm{~h}$. After $1 \mathrm{~h}$ the solution was taken out of the oven, allowed to cool to room temperature, and then $0.040 \mathrm{~g}$ of $\mathrm{H}_{4}$ TBApy was added into the vial. The mixture was sonicated until a homogeneous yellow suspension was obtained, which was then placed in an oven at $100^{\circ} \mathrm{C}$ for $24 \mathrm{~h}$. After $24 \mathrm{~h}$ the mother liquor was decanted. The resultant yellow powder was washed with $10 \mathrm{ml}$ of DMF three times over the course of $24 \mathrm{~h}$. Centrifugation (7,000 r.p.m. for $5 \mathrm{~min}$ in Eppendorf tubes) was used to separate the liquid and solid phases in between washings. The solid was re-suspended in $12 \mathrm{ml}$ of DMF and $0.5 \mathrm{ml}$ of $8 \mathrm{M} \mathrm{HCl}$ was added to the solution and swirled. The vial was placed in an oven at $100^{\circ} \mathrm{C}$ for $18 \mathrm{~h}$ and then removed. The mother liquor was decanted and the yellow powder was washed three times with $10 \mathrm{ml}$ of DMF over the course of $24 \mathrm{~h}$. The sample was then washed with $10 \mathrm{ml}$ of acetone three times over the course of $24 \mathrm{~h}$. Before physical measurements the samples were activated under dynamic vacuum at $120^{\circ} \mathrm{C}$ until a vacuum level of $\leq 0.002 \mathrm{~mm} \mathrm{Hg} \mathrm{min}^{-1}$ was reached.

Hydrolysis of DMNP. Hydrolysis experiments were carried out at room temperature. A solid sample of NU-1000 or NU-1000-dehyd (3.0 mg, $6 \mathrm{~mol} \%$, $0.0015 \mathrm{mmol} \mathrm{Zr}_{6}$ ) was added to an aqueous buffered solution containing $N$-ethylmorpholine $(1 \mathrm{ml}, 0.45 \mathrm{M})$ in a $1.5 \mathrm{ml}$ Eppendorf vial. The resulting mixture was stirred for $15 \mathrm{~min}$ to finely disperse the MOF powder. DMNP ( $4.0 \mu \mathrm{l}$, 
about $6.2 \mathrm{mg}, 0.025 \mathrm{mmol}$ ) was then added to this suspension. Periodic monitoring of the hydrolysis reaction was carried out by removing a $20 \mu \mathrm{l}$ aliquot from the reaction mixture and diluting it with an aqueous solution of $N$-ethylmorpholine $(10 \mathrm{ml}, 0.15 \mathrm{M})$ before ultraviolet-visible measurements Progress of the reaction was monitored by following the $p$-nitrophenoxide absorbance at $407 \mathrm{~nm}$, thus avoiding overlapping absorption bands from other species (for example, DMNP).

Monitoring the decomposition of GD in the presence of buffer. $3.3 \mathrm{mg}$ of NU-1000 was added to a 5-mm NMR tube followed by $47 \mu$ of ethylmorpholine $(0.5 \mathrm{M})$ and $0.7 \mathrm{ml}$ of distilled water, without shaking. Subsequently $2.6 \mu \mathrm{l}$ of GD was added to the same NMR tube and the sample was capped and shaken vigorously to mix. Caution! Experiments using GD should be run by trained personnel using appropriate safety procedures only. The sample was immediately placed into a Varian INOVA 400 NMR spectrometer and ${ }^{31} \mathrm{P}$ NMR spectra were taken (unlocked, with proton decoupling) over time to monitor the reaction: 64 scans per spectrum using $25^{\circ}$ pulses, a 0.5 -s acquisition time, and 3.25-s delay time between scans. Spectra were referenced to external $\mathrm{H}_{3} \mathrm{PO}_{4}(0 \mathrm{ppm})$.

Monitoring the decomposition of GD in the absence of buffer. To start, $30.9 \mathrm{mg}$ of NU-1000 was allowed to hydrate by exposure to $50 \% \mathrm{RH}$ air at $25^{\circ} \mathrm{C}$ then loaded into a 7-mm MAS NMR rotor by volume. In situ reactions were initiated by adding $5 \mu \mathrm{l}$ of neat agent to the MOF, and the rotor was then sealed with double o-ring caps and the removal was monitored with ${ }^{31} \mathrm{P}$ NMR over time. A Varian INOVA 400 NMR spectrometer equipped with a DOTY Scientific SuperSonic 7-mm VT-MAS probe was used for the experiments. 128 scans were performed using a $30^{\circ}$ pulse and $4 \mathrm{~s}$ delay between scans. Spectra were referenced to an external $85 \% \mathrm{H}_{3} \mathrm{PO}_{4}\left(0 \mathrm{ppm},{ }^{31} \mathrm{P}\right)$ standard.

Computational methods for studies with DMNP. Calculations were run with Gaussian 09 Rev. D.01 unless otherwise stated, using the M06-L functional and the def2-SVP basis set for all atoms except for $\mathrm{Zr}$, which was described by the SDD effective core potential and pseudopotential. Auto fitting was used for the basis set and an ultrafine grid was used for the two electron integrals. Geometry optimizations were run in the gas phase.

Computational methods for studies with GD and VX analogues. Geometry optimizations were performed for all species at the M06-L level of density functional theory. For the $\mathrm{Zr}$ atoms, the Stuttgart-Dresden (SDD) ECP28MWB basis set and associated pseudopotentials were used; the def2-SVP basis was used for $\mathrm{C}, \mathrm{O}, \mathrm{S}, \mathrm{P}$ and $\mathrm{H}$ atoms. The nature of stationary points was assessed in all cases by computation of analytic vibrational frequencies, which were also used to compute the molecular partition functions necessary to predict $298 \mathrm{~K}$ thermochemical quantities using the conventional ideal-gas, rigid-rotator, quantum-mechanical quasi-harmonic-oscillator approximation. Improved electronic energies were computed, as single-point calculations, using the same SDD basis set for Zr, but replacing def2-SVP with the def2-TZVPP basis for all other atoms.

\section{Received 10 December 2014; accepted 2 February 2015;} published online 16 March 2015

\section{References}

1. Raushel, F. M. Catalytic detoxification. Nature 469, 310-311 (2011).

2. Enserink, M. U. N. taps special labs to investigate Syrian attack. Science 341, 1050-1051 (2013).

3. Smith, B. M. Catalytic methods for the destruction of chemical warfare agents under ambient conditions. Chem. Soc. Rev. 37, 470-478 (2008).

4. Peterson, G. W. \& Rossin, J. A. Removal of chlorine gases from streams of air using reactive zirconium hydroxide based filtration media. Ind. Eng. Chem. Res 51, 2675-2681 (2012).

5. Wagner, G. W., Peterson, G. W. \& Mahle, J. J. Effect of adsorbed water and surface hydroxyls on the hydrolysis of VX, GD, and HD on titania materials: The development of self-decontaminating paints. Ind. Eng. Chem. Res. 51, 3598-3603 (2012).

6. Bandosz, T. J. et al. Reactions of VX, GD, and $\mathrm{HD}$ with $\mathrm{Zr}(\mathrm{OH})_{4}$ : Near instantaneous decontamination of VX. J. Phys. Chem. C 116, 11606-11614 (2012).
7. Furukawa, H., Cordova, K. E., O'Keeffe, M. \& Yaghi, O. M. The chemistry and applications of metal-organic frameworks. Science 341, 1230444 (2013).

8. Lee, J. et al. Metal-organic framework materials as catalysts. Chem. Soc. Rev. 38, 1450-1459 (2009).

9. Decoste, J. B. \& Peterson, G. W. Metal-organic frameworks for air purification of toxic chemicals. Chem. Rev. 114, 5695-5727 (2014).

10. Katz, M. J. et al. Simple and compelling biomimetic metal-organic framework catalyst for the degradation of nerve agent simulants. Angew. Chem. Int. Ed. 53, 497-501 (2013)

11. Cavka, J. H. et al. A new zirconium inorganic building brick forming metal organic frameworks with exceptional stability. J. Am. Chem. Soc. 130, 13850-13851 (2008)

12. Wu, H., Yildirim, T. \& Zhou, W. Exceptional mechanical stability of highly porous zirconium metal-organic framework UiO-66 and its important implications. J. Phys. Chem. Lett. 4, 925-930 (2013).

13. Mondloch, J. E. et al. Are $\mathrm{Zr}_{6}$-based MOFs water stable? Linker hydrolysis vs. capillary-force-driven channel collapse. Chem. Commun. 50, 8944-8946 (2014)

14. Mondloch, J. E. et al. Vapor-phase metalation by atomic layer deposition in a metal-organic framework. J. Am. Chem. Soc. 135, 10294-10297 (2013).

15. Planas, N. et al. Defining the proton topology of the $\mathrm{Zr}_{6}$-based metal-organic framework NU-1000. J. Phys. Chem. Lett. 5, 3716-3723 (2014).

16. Kickelbick, G., Wiede, P. \& Schubert, U. Variations in capping the $\mathrm{Zr}_{6} \mathrm{O}_{4}(\mathrm{OH})_{4}$ cluster core. X-ray structure analyses of $\left[\mathrm{Zr}_{6}(\mathrm{OH})_{4} \mathrm{O}_{4}\left(\mathrm{OOC}-\mathrm{CH}=\mathrm{CH}_{2}\right)_{10}\right]_{2}$ $\left(\mu-\mathrm{OOC}-\mathrm{CH}=\mathrm{CH}_{2}\right)_{4}$ and $\mathrm{Zr}_{6}(\mathrm{OH})_{4} \mathrm{O}_{4}(\mathrm{OOCR})_{12}(\mathrm{PrOH})(\mathrm{R}=\mathrm{Ph}, \mathrm{CMe}=$ $\mathrm{CH}_{2}$ ). Inorg. Chim. Acta 284, 1-7 (1999)

17. Rai, N. \& Siepmann, J. I. Transferable potentials for phase equilibria. 9. Explicit hydrogen description of benzene and five-membered and six-membered heterocyclic aromatic compounds. J. Phys. Chem. B 111, 10790-10799 (2007).

18. Chen, S-L., Fang, W-H. \& Himo, F. Theoretical study of the phosphotriesterase reaction mechanism. J. Phys. Chem. B 111, 1253-1255 (2007)

19. Parkin, G. Synthetic analogues relevant to the structure and function of zinc enzymes. Chem. Rev. 104, 699-768 (2004).

20. Wang, S., Bromberg, L., Schreuder-Gibson, H. \& Hatton, T. A. Organophophorous ester degradation by chromium(III) terephthalate metal-organic framework (MIL-101) chelated to $N, N$-dimethylaminopyridine and related aminopyridines. ACS Appl. Mater. Interfaces 5, 1269-1278 (2013).

21. Peterson, G. W. \& Wagner, G. W. Detoxification of chemical warfare agents by CuBTC. J. Porous Mater. 21, 121-126 (2014).

22. Dang, D. et al. Structural and catalytic performance of a polyoxometalate-based metal-organic framework having a lanthanide nanocage as a secondary building block. Inorg. Chem. 49, 1280-1282 (2010).

\section{Acknowledgements}

O.K.F., R.Q.S. and J.T.H. gratefully acknowledge DTRA for financial support (gran HDTRA-1-10-0023). C.J.C. gratefully acknowledges funding from the US DOE, Office of Basic Energy Sciences, Division of Chemical Sciences, Geosciences and Biosciences (Award DE-FG02-12ER16362). R.Q.S. acknowledges the National Energy Research Scientific Computing Center (NERSC) for computational resources. J.B.D. and G.W.P. gratefully acknowledge Joint Science and Technology Office for Chemical Biological Defense (JSTO-CBD) for funding (Project Number BA13PHM210).

\section{Author contributions}

J.E.M., M.J.K., J.T.H. and O.K.F. developed the concept. J.E.M., M.J.K. and W.B. carried out the materials synthesis, characterization, and catalysis with the simulant DMNP under the supervision of O.K.F. and J.T.H.; P.G. and P.L. carried out DFT simulations with DMNP under the supervision of R.Q.S.; W.C.I.III carried out DFT simulations with GD and VX under the supervision of C.J.C.; G.W.W. and M.G.H. carried out GD NMR degradation experiments under the guidance of J.B.D. and G.W.P.; J.E.M., M.J.K., J.T.H., and O.K.F. wrote the manuscript first draft and the rest of the authors contributed to the editing.

\section{Additional information}

Supplementary information is available in the online version of the paper. Reprints and permissions information is available online at www.nature.com/reprints. Correspondence and requests for materials should be addressed to J.T.H. or O.K.F.

\section{Competing financial interests}

R.Q.S., J.T.H. and O.K.F. have a financial interest in the start-up company NuMat Technologies, which is seeking to commercialize metal-organic frameworks. 